\title{
Prospective Randomized Comparative Study between Tension Band and Intramedullary Screw Fixation for Olecranon Fractures
}

\author{
Ahmed Sleem, Mohamed A. Mohamed, Abdelrahman H. Khalifa, Ahmed R. Abdelatif*, Ashraf Marzouk \\ Department of Orthopedics, Faculty of Medicine, Sohag University, Egypt \\ *Corresponding author: Ahmed R. Abdelatif, Mobile: (+20)1011856244, Email: ramadona_orthopedist@yahoo.com
}

\begin{abstract}
Background: Controversy exists regarding the best technique for treating displaced transverse olecranon fractures with advocates for three techniques: (1) intramedullary (IM) screw, (2) locking plate, and (3) tension band. Objectives: The aim of this study was to seek which method of fixation fits best according to olecranon fracture type and postoperative outcome. Two methods were used: K-wire with tension band and intramedullary screw.

Patients and methods: A prospective randomized comparative study included 20 patients with displaced transverse or oblique fracture of the olecranon, treated at Department of Orthopedics, Sohag University Hospital. The patients were randomly divided into two groups of equal number where patients with odd numbers were treated using $\mathrm{AO}$ tension band wiring (Group I) and those with even numbers by intramedullary screw (Group II).

Results: Patients' characteristics were insignificantly different between both groups. The range of motion was significantly higher in group II than group I. Mayo Elbow Performance score (MEPS) was significantly higher at 4 weeks, 3month and 6 months in group II compared to group I but with an insignificant difference at 2 weeks between both groups. Visual analogue score (VAS) was significantly lower at 6 months in group II compared to group I but with an insignificant difference at 2 weeks, 4 weeks and 3 month between both groups.

Conclusion: It could be concluded that olecranon fracture fixation with intramedullary screw is superior to K-wire with tension band in transverse or oblique non comminuted fractures with more range of motion, lower VAS, and higher MEPS.
\end{abstract}

Keywords: Tension Band, Intramedullary Screw Fixation, Olecranon Fractures.

\section{INTRODUCTION}

The olecranon is the proximal articular portion of the ulna. Along with the coronoid process, it forms the greater sigmoid notch which articulates with the humerus to provide flexion and extension of the elbow. Fractures of the olecranon are a relatively common injury in adults; its subcutaneous location leaves it vulnerable to injury from a direct blow. Fractures sustained range from simple non-displaced fractures to complex open fracture dislocations. Olecranon fractures frequently require surgical management to restore articular congruity and elbow stability ${ }^{(\mathbf{1})}$.

Most olecranon fractures follow low-energy trauma such as a fall from a height of less than 2 meters, a direct blow to the elbow, or from forced hyperextension. A fall on a partially flexed elbow may generate an avulsion fracture of the olecranon from the pull of the triceps. The fractures are usually isolated but associated lesions can occur in complex injuries and polytrauma cases ${ }^{(2)}$.

The fixation should be stable, it should allow an active elbow flexion and extension and it should promote union of the fracture. In the past, closed reduction and a plaster cast application was the treatment for fractures of the olecranon. But, a prolonged immobilization, with its own complications, increased the morbidity and the mortality of the patients. So, considering this, it has become important to intervene surgically. The active mobilization after surgery will restore the patient to normal functions as early as possible. The early and active movement not only prevents the tissue from fracture disease, but it greatly influences the quality and the rapidity of the fracture union ${ }^{(3)}$.

Many methods which have been described are tension band wiring, interfragmentary screws with or without wires, wires alone, plates, rush pin with tension band wiring, intramedullary screws with or without tension bands and bone fragment excision with reattachment triceps ${ }^{(4)}$.

AO tension band wiring is the most common method for fixation of these fractures; it involves the use of tension band and two Kirschner wires. Although this method has generally good results, it is not free of complications. The most common of these are hardware prominence requiring removal; other complications include loss of motion and loss of fixation. The K-wire, which is used in the AO tension band technique, resists the shear better than the figure of eight wire alone, but, it does not add compression to the fixation strength ${ }^{(5)}$.

Utilization of intramedullary screw fixation for olecranon fracture reduction has the advantage of decreasing irritation to surrounding tissues, while providing stability to facilitate early range of motion. This technique allows for rigid, anatomic fixation of the articular surface by anchoring fixation at the isthmus with the distal screw threads which provides the strength of fixation by converting the tensile force to a compressive force at the fracture site, with additional

This article is an open access article distributed under the terms and conditions of the Creative

Commons Attribution (CC BY-SA) license (http://creativecommons.org/licenses/by/4.0/) 
resistance to the displacement due to the lag screw compression $^{(6)}$.

The aim of this study was to seek which method of fixation fits best according to olecranon fracture type and postoperative outcome. Two methods were used: K-wire with tension band and intramedullary screw.

\section{PATIENTS AND METHODS}

This prospective randomized comparative study included a total of 20 patients with displaced transverse or oblique fracture of the olecranon, treated at Department of Orthopedics, Sohag University Hospital.

\section{Ethical approval:}

This study was conducted between June 2019 till June 2020, after taking ethical committee clearance from Sohag University hospital ethical board.Written informed consent of all the subjects was obtained.

The included subjects were divided randomly into two groups of equal number; Group 1, consisted of odd numbered patients treated by $\mathrm{AO}$ tension band wiring and Group 2 consisted of even numbered patients treated by intramedullary screw. All patients completed the follow up program.

\section{Inclusion criteria:}

- Adults.

- Transverse or oblique fractures.

- No comminution AO classification (21-A1) (21-B1) (Mayo type IA and IIA).

\section{Exclusion criteria:}

- Compound fractures

- Comminuted fractures.

- Monteggia fractures.

- Fracture- dislocations.

- Oblique fractures that extend distal to coronoid.

- Associated distal humeral fractures.

- Fractures with neurovascular deficits.

Preoperative clinical evaluation: Detailed history taking regarding mechanism of injury which may be road traffic accident, direct blow or fall from height.

Preoperative Radiological evaluation: We obtained anteroposterior and lateral radiographs of the elbows which were adequate for routine diagnosis and surgical planning. It was essential to obtain a true lateral radiograph of the elbow to evaluate the degree of displacement and articular comminution.
Classification: The Mayo classification of olecranon fractures were used.

Scoring: We used Mayo Elbow Performance Score (MEPS) ${ }^{(7)}$ and Visual Analogue Scale (VAS) ${ }^{(8)}$ for evaluation of the pain and function outcome to all patients.

Operative technique: The patient was positioned either supine with the arm across the chest or in the lateral decubitus with the arm draped over a padded support, allowing for the elbow to be moved from $90^{\circ}$ of flexion to full extension. General anesthesia was used.

1) Tension band wiring: A midline posterior skin incision and posterior approach was used extending one inch above the olecranon and two inches below. After cleaning of the fracture fragments and adequate irrigation of the joint with sterile saline, reduction of the fragments was done and maintained using reduction forceps with points and the adequacy of reduction was checked under image intensifier to ensure anatomical restoration of the articular surface. Two parallel Kirschner wires were then passed through the fracture site and through the anterior cortex aiming distal to coronoid process. A $2.5 \mathrm{~mm}$ hole was drilled transversely across the ulna distal to fracture site, an18gauge was then passed through the holes and placed in a figure-of-eight configuration. The proximal part of the wire was passed through the insertion of the triceps and around the K-wires. The wires were tensioned with one loop in the figure-of-eight configuration.

2) Intramedullary screw fixation: Using reduction forceps with points percutaneously and the adequacy of reduction was checked under image intensifier to ensure anatomical restoration of the articular surface. Provisional fixation is obtained with two parallel bicortical $1.0 \mathrm{~mm}$ k-wires that are placed in such a way that they will not hinder the placement of the cannulated intramedullary screw. We use two k-wires for provisional fixation to prevent rotation of the proximal ulna during placement of the screw. Placement of an intramedullary $2.8 \mathrm{~mm}$ guide wire over the centrecentre point of the palpable olecranon tip (Fig. 1). Screw length is then measured such that the distal threaded end of the screw will engage the narrow medulla of the proximal ulnar diaphysis (typically 90$110 \mathrm{~mm}$ ) to provide stable fixation. An incision about $0.5 \mathrm{~cm}$ is made around $2.8 \mathrm{~mm}$ guide wire. The cortex is opened with the cannulated drill and the screw is placed. An additional washer can be used. The guidewire and the two anti-rotation k-wires are then removed (Fig. 2). 


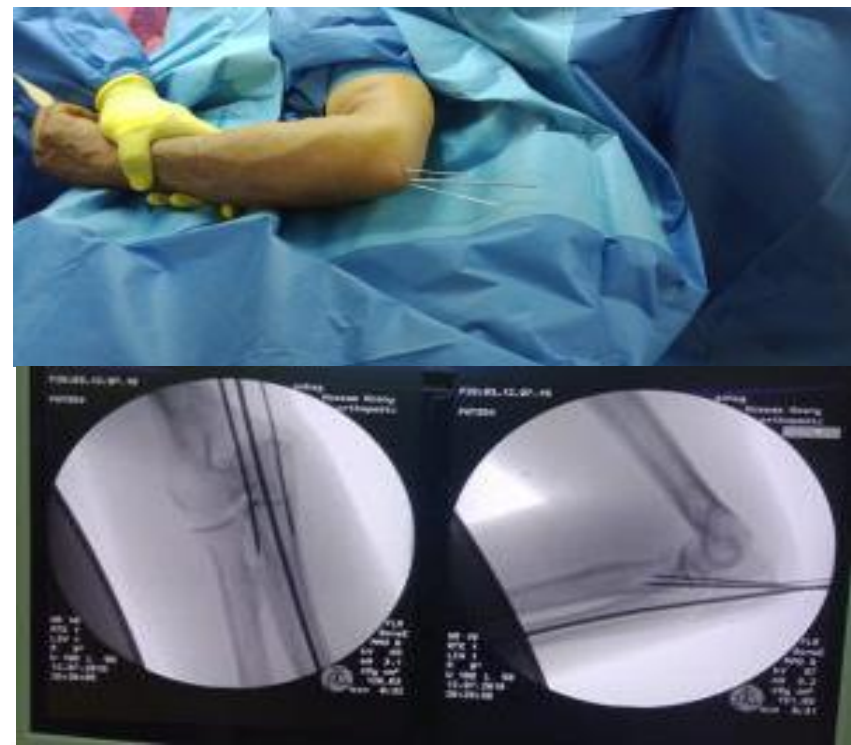

Figure (1): Provisional fixation is obtained with two parallel bicortical $1.0 \mathrm{~mm} \mathrm{k}$-wires then placement of an intramedullary $2.8 \mathrm{~mm}$ guide wire.
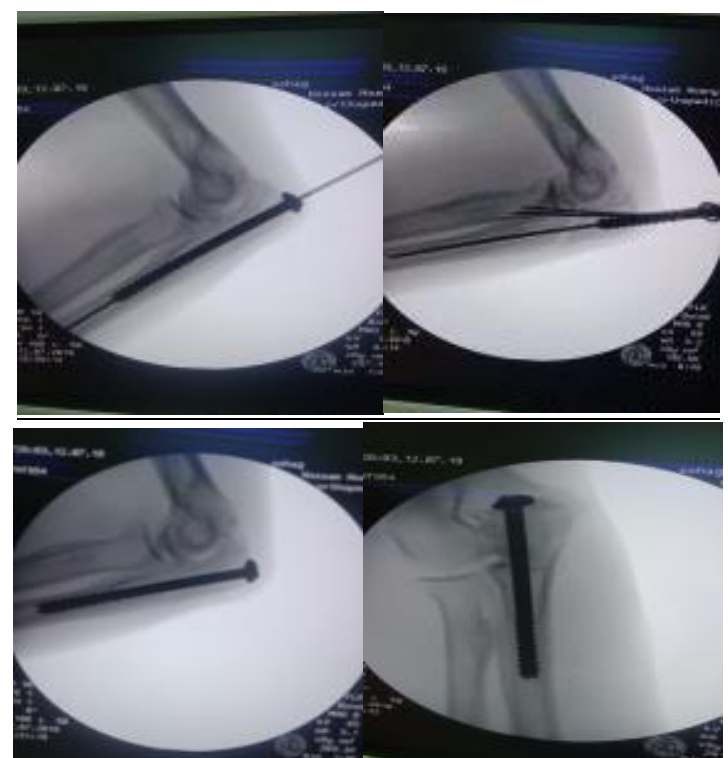

Figure (2): The cortex is opened with the cannulated drill and the screw is placed. An additional washer can be used. The guidewire and the two anti-rotation kwires are then removed.

\section{Postoperative management}

Patients were discharged and followed up in our outpatient clinic. In each follow up visit, the wound was inspected. The active elbow range of flexion and extension as well as pain scores and function according to Mayo elbow scoring system. Radiographic control with anteroposterior and lateral radiographs were obtained at 6 weeks, 3 and 6 months postoperatively to assess healing. All patients had broad arm sling arm sling immobilization for 7 to 10 days. By 2 weeks, gentle active assisted ROM exercises. By 6 weeks, light resistive exercises. By 3 months, elbow strengthening exercises

\section{Statistical analysis}

Statistical analysis was done by SPSS v25 (IBM Inc., Chicago, IL, USA). Numerical variables were presented as mean and standard deviation (SD) and were compared between the two groups utilizing Student's t- test. Categorical variables were presented as frequency and percentage $(\%)$ and were analysed utilizing Fisher's exact test when appropriate. A two tailed $\mathrm{P}$ value $<0.05$ was considered significant.

\section{RESULTS}

The age ranged from $18-83$ years with a mean value $28.5 \pm 6.79$ years in group I and ranged from $16-$ 83 years with a mean value $42.7 \pm 23.49$ years in group II. There were $6(60 \%)$ male patients and $4(40 \%)$ female patients in group I and there were $5(50 \%)$ male patients and $5(50 \%)$ female patients in group II. Patients' characteristics (Age and sex) were insignificantly different between both groups $(\mathrm{P}=0.083$ and 1.00 respectively) (table 1 ).

Table (1): Patients' characteristics in both studied groups.

\begin{tabular}{|c|c|c|c|c|}
\hline \multicolumn{2}{|c|}{} & Group I (n = 10) & Group II (n = 10) & \multirow{2}{*}{ P value } \\
\hline \multirow{2}{*}{ Age (years) } & Mean \pm SD & $28.5 \pm 6.79$ & $42.7 \pm 23.49$ & \multirow{2}{*}{0.083} \\
\cline { 2 - 4 } \multirow{2}{*}{ Sex } & Range & $18-40$ & $16-83$ & \multirow{2}{*}{1.00} \\
\cline { 2 - 4 } & Male & $6(60 \%)$ & $5(50 \%)$ & $5(50 \%)$ \\
\hline
\end{tabular}

As regard to the Mayo class, $4(40 \%)$ patients were 1A and $6(60 \%)$ patients were 2A in group I and 3 (30\%) patients were $1 \mathrm{~A}$ and $7(70 \%)$ patients were $2 \mathrm{~A}$ in group II. The Mayo class was insignificantly different between both groups $(\mathrm{P}=1)($ table 2$)$.

Table (2): Mayo class in both groups.

\begin{tabular}{|c|c|c|c|c|}
\hline \multicolumn{2}{|c|}{} & Group I (n= 10) & Group II (n= 10) & \multirow{2}{*}{ P value } \\
\hline \multirow{2}{*}{ Mayo class } & $1 \mathrm{~A}$ & $4(40 \%)$ & $3(30 \%)$ & \multirow{2}{*}{1.00} \\
\cline { 2 - 4 } & $2 \mathrm{~A}$ & $6(60 \%)$ & $7(70 \%)$ & \\
\hline
\end{tabular}

The range of motion ranged from $90-145$ with a mean value $126.5 \pm 16.51$ in group I and ranged from $120-160$ with a mean value $148 \pm 12.06$ in group II. The range of motion was significantly higher in group II than group I (P $=1)($ table 3$)$. 
Table (3): Range of motion in both groups.

\begin{tabular}{|c|c|c|c|c|}
\hline \multicolumn{2}{|l|}{} & $\begin{array}{c}\text { Group I } \\
(\mathbf{n = 1 0})\end{array}$ & $\begin{array}{c}\text { Group II } \\
(\mathbf{n}=\mathbf{1 0})\end{array}$ & P value \\
\hline Range of motion & Mean \pm SD & $126.5 \pm 16.51$ & $148 \pm 12.06$ & $0.004 *$ \\
\hline
\end{tabular}

* significant as $\mathrm{P}$ value $<0.05$

Mayo Elbow Performance score (MEPS) was significantly higher at 4weeks, 3month and 6month in group II compared to group I ( $\mathrm{P}$ value $=0.032,0.042$ and 0.031 respectively) but with an insignificant difference at 2 weeks between both groups $(\mathrm{P}$ value $=0.0501)($ table 4$)$.

Table (4): Mayo Elbow Performance score (MEPS) in both groups.

\begin{tabular}{|c|c|c|c|c|c|}
\hline & & 2 weeks & 4 weeks & 3 month & 6 month \\
\hline \multirow{2}{*}{$\begin{array}{l}\text { Group I } \\
(\mathbf{n}=\mathbf{1 0})\end{array}$} & \multirow{2}{*}{ Mean \pm SD } & 71.5 Fair & 75 Good & 80 Good & 80Good \\
\hline & & 7.84 & 9.13 & 9.13 & 9.13 \\
\hline \multirow{2}{*}{$\begin{array}{c}\begin{array}{c}\text { Group II } \\
(\mathbf{n}=\mathbf{1 0})\end{array} \\
\end{array}$} & \multirow{2}{*}{ Mean \pm SD } & 78 Good & 83.5 Good & 88.5 Good & 89 Good \\
\hline & & 5.87 & 7.09 & 8.18 & 8.10 \\
\hline \multicolumn{2}{|c|}{ P value } & 0.0501 & 0.032* & $0.042 *$ & 0.031* \\
\hline
\end{tabular}

Visual analogue score (VAS) was significantly lower at 6 month in group II compared to group I (P value $=0.030)$ but with an insignificant difference at 2 weeks, 4 weeks and 3 month between both groups ( $\mathrm{P}$ value $=0.472,0.209$ and 0.660 respectively) (table 5).

Table (5): Visual analogue score (VAS) in both groups.

\begin{tabular}{|c|c|c|c|c|c|}
\hline & & 2 weeks & 4 weeks & 3 month & 6 month \\
\hline \multirow{2}{*}{$\begin{array}{l}\text { Group I } \\
(\mathbf{n}=\mathbf{1 0})\end{array}$} & \multirow{2}{*}{ Mean \pm SD } & 6.40 & 6.90 & 5.20 & 4.50 \\
\hline & & 1.71 & 1.66 & 1.48 & 1.65 \\
\hline \multirow{2}{*}{$\begin{array}{c}\begin{array}{c}\text { Group II } \\
(\mathbf{n}=\mathbf{1 0})\end{array} \\
\end{array}$} & \multirow{2}{*}{ Mean \pm SD } & 5.80 & 6.00 & 4.90 & 2.70 \\
\hline & & 1.93 & 1.41 & 1.52 & 1.77 \\
\hline \multicolumn{2}{|c|}{$P$ value } & 0.472 & 0.209 & 0.660 & $0.030 *$ \\
\hline
\end{tabular}

Union in both groups: There were (100\%) patients with union and no patients with non-union in both groups.

Hardware removal in both groups: As regard to hardware removal, no patients were removed the hardware in both groups.

Complications in both groups: There was one patient (10\%) developed complications (hardware irritation and pain) and $9(90 \%)$ patients without complications in group I and all patients (100\%) were without complications in group II.

\section{DISCUSSION}

Olecranon fractures are relatively common injuries and account for approximately $10 \%$ of upper extremity fractures in adults. Traction of the triceps on the proximal fragment often leads to disruption of articular congruity and of the elbow's extension mechanism. As a result, this injury is typically treated with open reduction and internal fixation (ORIF). Common techniques to treat simple olecranon fractures are tension-band wiring and plate fixation ${ }^{(9)}$. As the skin is thin at the proximal ulna with relatively little subcutaneous tissue, these fixation methods often lead to implant-related soft-tissue irritation necessitating implant removal in $68-82 \%$ of the cases largely based on the fixation method that was used ${ }^{(\mathbf{1 0})}$.

While intramedullary screw fixation is commonly used to fix olecranon osteotomies. Fewer reports have been published on its use for simple (Mayo type I or IIA) olecranon fractures.
In the current study, regarding to the patients' characteristics, age and sex were insignificantly different between both two groups. The affected side, The Mayo class, the mechanism of injury and complications were insignificantly different between both groups. The range of motion was significantly higher in group II than group I $(\mathrm{P}=0.004)$.

Visual analogue score (VAS) was significantly lower at 6 months in group II compared to group I but with an insignificant difference at 2 weeks, 4 weeks and 3month between both groups. Mayo Elbow Performance score (MEPS) was significantly higher at 4weeks, 3 months and 6month in group II compared to group I but with an insignificant difference at 2 weeks between both groups.

In agreement with our results, Hutchinson et al. (11) studied ten cadaveric elbows with use of cyclic loading that simulated active range of motion and pushing up from a chair. Each specimen underwent 
fixation of a simulated $50 \%$ transverse olecranon fracture with use of intramedullary and cortically fixed tension band motion, lower VAS and higher MEPS. Constructs followed by fixation with a $7.3-\mathrm{mm}$ diameter cancellous screw with and without a tension band. They demonstrated that cancellous screw provided the most stable fixation than that provided by the Kirschner-wire techniques. Use of the tension band in conjunction with the intramedullary screw improved the stability of fixation.

In agreement with our results, Molloy et al. ${ }^{(12)}$ compared the biomechanical stability of an alternative custom-designed intramedullary nail (IMN) fixation with that of traditional tension band wiring (TBW), the gold standard for stabilizing transverse olecranon fractures. They demonstrated that the locked IMN provided stronger and stiffer fixation than did TBW. Theoretically the IMN fixation would require less surgical exposure and would be expected to require fewer revisions than TBW fixation.

In agreement with our results, Bosman et al. ${ }^{(13)}$ identified 15 patients (average age at index procedure 44 years, range 16-83) with a Mayo type I or IIA olecranon fracture who were treated with an intramedullary cannulated screw. They demonstrated that patients had complete union of their fracture and 1 patient had an asymptomatic non-union that did not require further intervention. Average flexion was $145^{\circ}$ (range 135-160) and the average extension lag was $11^{\circ}$ (range 0-30) so Fixation of simple olecranon fractures with an intramedullary screw is a safe and easy fixation method in young patients, leading to good functional and radiological results.

Malreduction of a simulated olecranon fracture was most significant when the starting point for the IM screw was malpositioned medially. A central or laterally based starting point was more forgiving. Avoiding a medially based starting point is crucial for achieving benefits of fixation with an IM screw and reduces the chance of malreduction after fixation ${ }^{(\mathbf{1 4})}$.

Our present study had: some points of strengths that operation was done by the same surgical team, the results were analyzed by an independent investigator to avoid investigator bias and the clinical and functional outcome was determined based on standard scores while the points of weakness were the relatively small sample size.

\section{CONCLUSION}

It could be concluded that olecranon fracture fixation with intramedullary screw is superior to K- wire with tension band in transverse or oblique non comminuted fractures with more range of motion, lower VAS and higher MEPS.

\section{REFERENCES}

1. Sullivan C, Herron T, Hayat $Z$ (2020): Olecranon Fracture. In: StatPearls [Internet]. Treasure Island (FL): StatPearls https://pubmed.ncbi.nlm.nih.gov/30725980/

Publishing.

2. Bailey C, MacDermid J, Patterson S et al. (2001): Outcome of plate fixation of olecranon fractures. Journal of Orthopaedic Trauma, 15(8): 542-548.

3. Raju S, Gaddagi R (2013): Cancellous screw with tension band wiring for fractures of the olecranon. Journal of Clinical and Diagnostic Research, 7(2): 339-43.

4. Hak D, Golladay G (2000): Olecranon fractures: treatment options. Journal of the American Academy of Orthopaedic Surgeons, 8(4): 266-275.

5. Heim U, Pfeiffer K (2012): Small fragment set manual: technique recommended by the ASIF Group. Springer Science \& Business Media. https://www.springer.com/gp/book/9783642966729

6. Harmon $P$ (1945): Treatment of fractures of the olecranon by fixation with stainless-steel screws. JBJS., 27(2): 328-329.

7. Dawson J, Fitzpatrick R, Carr A et al. (1996): Questionnaire on the perceptions of patients about total hip replacement. The Journal of Bone and Joint Surgery, 78(2): 185-190.

8. Gummesson C, Atroshi I, Ekdahl C (2003): The disabilities of the arm, shoulder and hand (DASH) outcome questionnaire: longitudinal construct validity and measuring self-rated health change after surgery. BMC Musculoskeletal Disorders, 4(1): 11-16.

9. Matar H, Ali A, Buckley S et al. (2014): Surgical interventions for treating fractures of the olecranon in adults. Cochrane Database Syst Rev., 11: Cd010144.

10. Duckworth A, Clement N, White T et al. (2017): Plate versus tension-band wire fixation for olecranon fractures: a prospective randomized trial. JBJS., 99(15): 1261-73

11. Hutchinson D, Horwitz D, Ha G et al. (2003): Cyclic loading of olecranon fracture fixation constructs. JBJS., 85(5): 831-7.

12. Molloy S, Jasper L, Elliott D et al. (2004): Biomechanical Evaluation of Intramedullary Nail Versus Tension Band Fixation for Transverse Olecranon Fractures. Orthopaedic Trauma, 18(3): 170-4.

13. Bosman W, Emmink B, Bhashyam A et al. (2020): Intramedullary screw fixation for simple displaced olecranon fractures. European Journal of Trauma and Emergency Surgery, 46(1): 83-9.

14. Potter G, Mascarenhas D, Sciadini M et al. (2018): What is the ideal starting point for an olecranon screw? An anatomic cadaveric study. Journal of Orthopaedic Trauma, 32(6): 313-319. 